\title{
P-Selectin Imaging in Cardiovascular Disease: What You See Is What You Get?
}

$\mathbf{I}_{\mathrm{n}}$ nflammation and hemostasis are key processes in the pathogenesis of atherosclerotic cardiovascular disease $(1,2)$. Vascular inflammation in response to lipid depositions causes formation of atherosclerotic plaques (1). In addition, inflammation in existing plaques is the primary process responsible for progression toward a phenotype that is vulnerable to rupture and thereby causing acute events such as acute myocardial infarction and stroke $(1,3,4)$. Plaque rupture can trigger the hemostatic system, which once activated generates intraplaque and intravascular platelet thrombi (2). Small nonocclusive thrombi contribute to

\section{See page 1433}

further increase of plaque vulnerability, whereas large occlusive thrombi are responsible for acute cardiovascular events (5).

Both inflammation and hemostasis are characterized by changed interactions between activated endothelial cells, platelets, and leukocytes. Activation markers presented on the surfaces of these cells may be valuable imaging targets to detect these processes. One of the most promising among those is the adhesion molecule $\mathrm{P}$-selectin, which is presented on activated platelets and leukocytes and

Received May 17, 2011; accepted Jun. 13, 2011.

For correspondence contact: Hendrikus $\mathrm{H}$. Boersma, Department of Hospital and Clinical Pharmacy EB 70, University of Groningen, University Medical Center Groningen, P.O. Box 30.001, NL-9700 RB Groningen, The Netherlands.

E-mail: h.h.boersma@umcg.nl

Published online Aug. 4, 2011.

COPYRIGHT ( 2011 by the Society of Nuclear Medicine, Inc.

DOI: 10.2967/jnumed.111.090506 plays a key mediating role in inflammatory and hemostatic cellular interactions (6).

In inflammation, P-selectin on endothelial cells and platelets results in tethering of leukocytes, followed by rolling over the endothelium, and subsequent migration into the tissue (6). Also, P-selectin on platelets mediates formation of platelet-leukocyte complexes, which enhance migration (6). Likewise, during vascular thrombus formation, platelet-leukocyte complexes are formed, causing growth and stabilization of the thrombus (5).

Since the discovery of the role of $P$-selectin in cardiovascular inflammation, several P-selectin imaging methods have been developed and explored. The feasibility of P-selectin imaging has been shown in the settings of early stage atherosclerosis using MRI $(7,8)$. Furthermore, myocardial ischemia and reperfusion were assessed using molecular ultrasound imaging (9). However, methodologic problems remain. The agents used were based either on P-selectin-avid antibodies $(10,11)$ or on synthetic ana$\operatorname{logs}$ of Sialyl Lewis ${ }^{\mathrm{X}}\left(\mathrm{SLe}^{\mathrm{X}}\right)$, the natural ligand of P-selectin $(7,8,10)$. Although the antibody-based agents generally yield good imaging results $(9,11)$, the production of these tracers is expensive and time-consuming. The clinical potential of $\mathrm{SLe}^{\mathrm{X}}$ analogs, on the other hand, is limited by their poor imaging properties $(7,8,10)$.

In this issue of The Journal of Nuclear Medicine, Rouzet et al. report the development of a novel and promising tracer for imaging P-selectin: ${ }^{99 \mathrm{~m} T c-f u c o i d a n ~(12) . ~ T h e y ~}$ used this tracer to show P-selectin upregulation in animal models of 2 different settings: myocardial ischemia- reperfusion injury and platelet-rich thrombus.

The described imaging method has several advantages over the previously developed imaging protocols. Notably, it has practical advantages, improving its potential to be used as a clinical agent in the future. First, the production of ${ }^{99 m} \mathrm{Tc}$-fucoidan is relatively simple. The fucoidan polysaccharide can be easily extracted from algae, and it can be labeled with ${ }^{99 \mathrm{~m}} \mathrm{Tc}$ using a standard reduction method. Also, a clinically utilizable imaging protocol of $45 \mathrm{~min}$ at $2 \mathrm{~h}$ after injection was used, yielding good imaging results. Mean target-to-background ratios were 3.6-5.1 (12). Moreover, ${ }^{99 m}$ Tc-fucoidan benefits from the wide clinical availability of SPECT scanners.

However, compared with the previously used MRI and ultrasound modalities, SPECT also has disadvantages. Most important, SPECT does not show great anatomic detail. And as P-selectin is implicated in various stages of atherosclerotic disease, anatomic information is necessary to determine which stage is being shown. Therefore, the addition of CT to SPECT would be a feasible option in most settings. Furthermore, because of the superior resolution of PET, and the current development of PET/MRI scanners, development and subsequent production of fucoidan-based PET tracers could further enhance the potential of P-selectin imaging.

Although the use of fucoidan appears to be safe for the clinical setting from a toxicologic and pharmacologic perspective $(9,11)$, the method used to isolate this tracer from algae does not as yet comply with the current standards of good manufacturing practice (GMP). Therefore, a standardized method of 
producing fucoidan compliant with good manufacturing practices, which guarantees a consistent-quality tracer, needs to be developed.

Because of the role of P-selectin in cardiovascular inflammation and hemostasis, a validated P-selectin imaging technique holds promise for imaging in several cardiovascular settings. Among these are screening of early atherosclerotic disease (12), detection of highrisk atherosclerotic lesions, detection of myocardial ischemia and reperfusion $(7,8)$, and evaluation of therapies for treatment of ischemic injury $(10,11)$. Early detection of disease activity can result in earlier change of lifestyle and start of treatment, thereby reducing the risk of atherosclerotic complications. Identification of vulnerable plaques could guide clinical decision making and patient selection for preventative intervention, reducing the numbers that need to be treated (9). The superiority of a P-selectin imaging protocol over other imaging protocols for similar purposes, such as the apoptotic tracer ${ }^{99 \mathrm{~m}} \mathrm{Tc}$-annexin A5 (13) and ${ }^{18} \mathrm{~F}-\mathrm{FDG}$, is yet to be proven (14).

In the diagnosis of acute coronary syndrome (15), P-selectin imaging may also play a role because the molecule is central in the inflammatory response triggered by ischemia. Diagnosis of acute coronary syndrome in patients in the emergency room remains an important challenge to clinicians. Current failure to identity all patients at risk results in high rates of costly false-positive admissions $(50 \%)$ and a considerable number of false-negative discharges from the emergency unit $(6 \%-7 \%)$. The latter results in complications and subsequent additional morbidity in the false-negative patients (11).

As P-selectin expression reflects ischemic stress, it could be a useful target to determine the clinical effect of new therapies in myocardial and cerebral ischemic injury (11).
A role for $\mathrm{P}$-selectin imaging in almost every stage of atherosclerotic disease could be envisioned. However, this could turn out to be a doubleedged sword, as it is questionable whether P-selectin imaging will prove to be selective enough. For instance, it remains to be determined whether this P-selectin imaging method can distinguish between vulnerable thrombotic plaques and plaques that are inflammatory and active but in an earlier stage of development and therefore relatively stable. Also, it is conceivable that $\mathrm{P}$-selectin imaging cannot easily detect coronary syndrome: the P-selectin already present on the atherosclerotic vasculature might outshine the ischemia-induced $\mathrm{P}$ selectin expression. In the animal models of acute events, no atherosclerotic vessels were present and vice versa $(8,12,16)$. Future studies should give additional attention to these issues.

P-selectin plays a central role in cardiovascular disease. The current radiopharmaceutical $99 \mathrm{~m} \mathrm{Tc}$-fucoidan may be of additional value to the other P-selectin imaging techniques that are currently under development. As 99m Tc-fucoidan and the other available imaging agents all have their advantages and disadvantages, it remains to be determined which one is most suitable for clinical use. Furthermore, it has to be determined whether P-selectin imaging can distinguish between the different stages of atherosclerotic disease.

\section{Hendrikus H. Boersma \\ University of Groningen \\ Groningen, The Netherlands}

Hans J. de Haas

University of Groningen

Groningen, The Netherlands

Chris P.M. Reutelingsperger

Maastricht University

Maastricht, The Netherlands
Riemer H.J.A. Slart

University of Groningen

Groningen, The Netherlands

\section{REFERENCES}

1. Libby P, Ridker PM, Maseri A. Inflammation and atherosclerosis. Circulation. 2002;105:1135-1143.

2. Borissoff JI, Spronk HM, ten Cate H. The hemostatic system as a modulator of atherosclerosis. $N$ Engl J Med. 2011;364:1746-1760

3. Emsley HC, Smith CJ, Tyrrell PJ, Hopkins SJ. Inflammation in acute ischemic stroke and its relevance to stroke critical care. Neurocrit Care. 2008;9:125-138.

4. Yellon DM, Hausenloy DJ. Myocardial reperfusion injury. N Engl J Med. 2007;357:1121-1135.

5. Mann J, Davies MJ. Mechanisms of progression in native coronary artery disease: role of healed plaque disruption. Heart. 1999;82:265-268.

6. Blann AD, Nadar SK, Lip GY. The adhesion molecule P-selectin and cardiovascular disease. Eur Heart J. 2003;24:2166-2179.

7. Jacobin-Valat MJ, Deramchia K, Mornet S, et al. MRI of inducible P-selectin expression in human activated platelets involved in the early stages of atherosclerosis. NMR Biomed. December 29, 2010 [Epub ahead of print].

8. McAteer MA, Schneider JE, Ali ZA, et al. Magnetic resonance imaging of endothelial adhesion molecules in mouse atherosclerosis using dualtargeted microparticles of iron oxide. Arterioscler Thromb Vasc Biol. 2008;28:77-83.

9. Barber PA, Foniok T, Kirk D, et al. MR molecular imaging of early endothelial activation in focal ischemia. Ann Neurol. 2004;56:116-120.

10. Kaufmann BA, Lewis C, Xie A, Mirza-Mohd A, Lindner JR. Detection of recent myocardial ischaemia by molecular imaging of P-selectin with targeted contrast echocardiography. Eur Heart J. 2007;28:2011-2017.

11. Villanueva FS, Lu E, Bowry S, et al. Myocardial ischemic memory imaging with molecular echocardiography. Circulation. 2007;115:345-352.

12. Rouzet F, Bachelet-Violette L, Alsac J, et al. Radiolabeled fucoidan as a P-selectin targeting agent for in vivo imaging of platelet-rich thrombus and endothelial activation. $J$ Nucl Med. 2011;52:1433-1440.

13. Hermus L, van Dam GM, Zeebregts CJ. Advanced carotid plaque imaging. Eur J Vasc Endovasc Surg. 2010;39:125-133.

14. Kolodgie FD, Petrov A, Virmani R, et al. Targeting of apoptotic macrophages and experimental atheroma with radiolabeled annexin V: a technique with potential for noninvasive imaging of vulnerable plaque. Circulation. 2003;108:3134-3139.

15. Ogawa $\mathrm{M}$, Ishino $\mathrm{S}$, Mukai $\mathrm{T}$, et al. ${ }^{18} \mathrm{~F}-\mathrm{FDG}$ accumulation in atherosclerotic plaques: immunohistochemical and PET imaging study. $\mathrm{J} \mathrm{Nucl}$ Med. 2004;45:1245-1250.

16. Downey JM, Cohen MV. Why do we still not have cardioprotective drugs? Circ J. 2009;73:1171-1177. 